PHILOSOPHY OF PROBABILITY 


\section{PHILOSOPHICAL STUDIES SERIES}

Founded by Wilfrid S. Sellars and Keith Lehrer

Editor:

KEITH LEHRER, University of Arizona

Board of Consulting Editors:

JONATHAN BENNETT, Syracuse University

ALLAN GIBBARD, University of Michigan

ROBERT STALNAKER, Massachusetts Institute of Technology

ROBERT G. TURNBULL, Ohio State University

VOLUME 56 


\title{
PHILOSOPHY OF PROBABILITY
}

\author{
Edited by \\ JACQUES-PAUL DUBUCS \\ University of Paris I - Sorbonne and C.N.R.S.
}

Springer-Science+Business Media, B.V . 
Library of Congress Cataloging-in-Publication Data

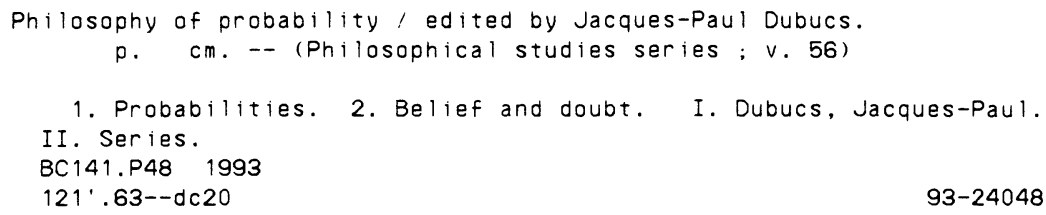

ISBN 978-90-481-4301-6 ISBN 978-94-015-8208-7 (eBook)

DOI 10.1007/978-94-015-8208-7

Printed on acid-free paper

\author{
All Rights Reserved \\ (C) 1993 Springer Science+Business Media Dordrecht
}

Originally published by Kluwer Academic Publishers in 1993.

Softcover reprint of the hardcover 1st edition 1993

No part of the material protected by this copyright notice may be reproduced or utilized in any form or by any means, electronic or mechanical, including photocopying, recording or by any information storage and retrieval system, without written permission from the copyright owner. 
CONTENTS

INTRODUCTION $\quad$ ix

CHAPTER 1: PERSONALISTIC BAYESIANISM

Colin Howson

CHAPTER 2: ON HIGHER ORDER BELIEFS

Nils-Eric Sahlin

1. Pros 13

2. Cons 23

3. A Historical Remark 26

CHAPTER 3: ON THE LOGIC OF RELEVANCE

Peter Gärdenfors $\quad 35$

1. Introduction 35

2. Basic Criteria for the Relevance

3. A Trivialization Result $\quad 40$

4. Two Further Criteria 42

5. The Conjunction Criterion for Relevance 43

6. The Conjunction Criterion for Irrelevance $\quad 45$

7. An Alternative Definition of
the Relevance Relation

$\begin{array}{ll}\text { 8. Conclusion } & 50\end{array}$

CHAPTER 4: DIVERGING DISTRIBUTIONS David Miller 55

1. The Logistic Function $\quad 55$

2. The Binomial Distribution 58

3. A Chaotic Distribution $\quad 59$

4. Another Chaotic Distribution 64

5. Scientific Objectivity 71

6. Approximate Distributions $\quad 72$

7. Conclusion $\quad 74$ 
vi

CHAPTER 5: INDUCTIVE LOGIC REVISITED

Jacques-Paul Dubucs

1. Carnap's System $\quad 80$

2. Inductive Logic in a Qualitative Setting 83

3. What is Inductive Support?

4. Appendix 105

CHAPTER 6: PROBABILITY AND UTILITY John M. Vickers 109

1. Utility 109

2. Action, Belief, and Desire 111

3. Adrift 114

4. The Limits of Subjectivity 119

5. Deliberation 121

6. On the Logic of Deliberation 123

7. Conclusion 125

CHAPTER 7: WHAT HAS PROBABILITY TO DO WITH STRENGTH OF BELIEF L. Jonathan Cohen 129

Section I 129

Section II $\quad 130$

Section III $\quad 132$

Section IV 133

Section V 135

Section VI 136

Section VII $\quad 138$

Section VIII 139

Section IX $\quad 141$

Section X 142

CHAPTER 8: RANDOMNESS, UNPREDICTABILITY AND

ABSENCE OF ORDER: THE IDENTIFICATION BY THE THEORY OF RECURSIVITY OF THE MATHEMATICAL NOTION OF RANDOM SEQUENCE Jean-Paul Delahaye 145

1. Abstract 145

2. Introduction 146

3. The Notion of Martin-Löf-Chaitin 
4. Comparison of Church-Turing's Thesis and Martin-Löf-Chaitin's Thesis

CHAPTER 9: A GLANCE AT NON-STANDARD MODELS AND LOGICS OF UNCERTAINTY AND VAGUENESS

Didier Dubois and Henri Prade

Introduction

1. Various Facets of Imperfect Information

2. Conditioning, Independence, Expectations and the Combination of Information

3. Non-Standard Logics of Uncertainty and Vagueness

4. Conclusion

CHAPTER 10: CAUSAL LAWS ARE OBJECTIFICATIONS OF INDUCTIVE SCHEMES Wolfgang Spohn

1. Is Causation Objective?

2. Induction 227

3. Causation 231

4. An Explication of Objectification 234

5. The Objectification of Induction and Causation 239

6. Outlook 249

CHAPTER 11: PROBABILISTIC INFERENCE IN ARTIFICIAL INTELLIGENCE: THE METHOD OF BAYESIAN NETWORKS Jean-Louis Golmard

1. Introduction 257

2. Knowledge Representation 259

3. Knowledge Utilization 264

4. Open Problems 283

5. Conclusions 285 


\section{INTRODUCTION}

1. Belief and probability. According to the subjectivistic view advocated by Ramsey and de Finetti, the probability of a sentence measures the strength of the belief in it. Probability is operationally defined in terms of the agent's willingness to take bets on the truth of the sentence: roughly stated, the probability of $E$ is the maximum amount of money that the agent is prepared to spend for subscribing to a contract that pays $\$ 1$ if $E$ is true and nothing otherwise. Thus the subjectivist justifies the classical laws of probability by showing that an agent who violates them (by acting on "incoherent" probabilities) is liable to unescapable loss: in such a case, an opponent could build a "Dutch book", i.e. a combination of bets that the agent would be committed to accept but which would lead him to a guaranteed loss.

Several objections have been raised to this view of probability. Two objections, which are discussed at length in Colin Howson's paper, are the following:

1) Subjectivism seems to postulate an unrealistic precision in measuring the strength of the beliefs of the agents. According to several authors (e.g. Kyburg), it would be preferable to represent degrees of belief by intervals whose endpoints are lower and upper probabilities.

2) The subjectivist framework does not provide any obvious way to cope with the reinforcements of the beliefs that arise from the mere improvement of the "quality" of evidence. For suppose an agent is given a coin which appears to be fair, so that he believes to the degree 0.5 that the coin will fall head when tossed. Now this degree surely remains unchanged after seeing 10,000 tosses of which 5000 give heads. But the mere indication of this punctual subjective probability does not suffice to reflect the additional information resulting from the observed sequence.

Both objections are not unrelated. For the improvement of the evidence in 2) can be mirrored by the contraction of the interval representing the belief assessment, if we express the strength of belief, as suggested in 1), by a probability interval rather than by a unique probability measure. But the question is not simply a technical one. For in elaborating such devices for representing the epistemic reliability of the basic probability assessments, we are fatally driven to beg the question of the 
very subjectivist account of the probability: if a probability assessment can be said to be more or less reliable, then probability is not merely a matter of personal degree of belief, but rather of something more akin to a rational degree of belief (not something that we have, but something that we ought to have). As for Nils-Eric Sahlin, he scrutinizes various strategies in dealing with the question of epistemic reliability, and shows that the socalled subjectivist school is divided on this point, and that Ramsey himself greatly differs from such extreme subjectivists as de Finetti or Savage by admitting that some probability assessments are, in some sense, more rational than others.

In order to account for the epistemic reliability of the probability assessments, we should moreover consider as relevant to a sentence some evidential items which do not alter the prior probability of the sentence. The standard sense of 'relevance' in the probability-theory setting, according to which an evidence $E$ is 'relevant' to $H$ if and only if it alters favourably or unfavourably the current probability of $\mathrm{H}$, is therefore to be rejected. But we have already other reasons to thrust it aside. For example Keynes has stressed that the whole evidence E\&F should always be considered as relevant to $H$ if $P(H / E) \neq P(H)$, even if $P(H / E \& F)=P(H)$. Keynes' own attempt to modify the definition of relevance accordingly has been shown by Carnap to be reducible to a triviality. Peter Gärdenfors tries in his paper to resolve the difficulty by defining a non trivializable notion of relevance which enlarges, conforming to Keynes' basic intuition, the set of the sentences that are relevant to a given sentence.

The difficulties related to epistemic reliability are by no means the only ones subjectivism has to face. Other objections can be raised against the subjectivist pretension to furnish a correct account of the objectivity of science. The basic subjectivist claim on this point concerns the explanation of the intersubjective agreement on the degrees of beliefs in the scientific community. According to the partisans of the doctrine, the observed consensus is the simple result of a process of learning by conditionalization: whatever the agents choose as their prior distributions, their posterior distributions will converge as soon as they accumulate the same evidence. In other words, differences in prior probabilities do not matter, for consensus is, in the long run, the inevitable and automatic result of the iterated process of conditionalization. David Miller's paper is devoted to 
a provocative attack against this pious image. It presents dramatic counterexamples to the rule of convergence of distributions: there are cases in which small variations in the priors lead to arbitrarily large variations in the posteriors, as well as cases in which increase of evidence leads to random fluctuations in them.

The logical tradition proposes a non-subjectivist account of the probabilistic assessments based on a balance of evidence, and its main objective is to determine in a purely formal way the degree to which a sentence is supported by an evidential corpus. Carnap thought that this goal could be reached by a suitable extension of the methods of deductive logic, and used probability to build a formal system concerned with inductive or sub-demonstrative inferences. Jacques-Paul Dubucs' paper deals with some well-known objections to Carnap-like constructions, and it tries to show that several of them can be dismissed if one renounces to the usual definition of inductive confirmation in terms of increasing probability.

The papers quoted so far are concerned with the use of probability in determining the impact of the available evidence on (rational) belief. But probability is too crucially involved in another kind of rationality, namely behavioral rationality, viewed as consistency between decisions, beliefs and desires. It is precisely the matter John Vickers is concerned with. $\mathrm{He}$ scrutinizes in his paper the significance of the pragmatic principle according to which we act in such a way that we believe that our actions will transform the actual world into a world in which our desires are more likely to be satisfied: he explores especially the variability of the meanings of this principle between objective and subjective interpretations, and proposes a logical way of grading it.

The subjectivist interpretation of the probability as measuring the strength of belief is deeply rooted, via the pragmatic principle, in the image of man that emerges from the so-called "folk-psychology". But L. Jonathan Cohen argues that this image, at least in the standard (humean) way of conceiving it, is not worth taking as a satisfactory foundation, neither for cognitive science, nor for the philosophy of probability. He proposes to replace (or to complete) the standard explanation of behavior as the result of an interaction between passive components (involuntary beliefs, recorded desires) by a cartesian explanation involving active factors (acceptation of premisses, adoption of goals). And he claims that such an alternative basis for cognitive science is also, precisely, what a correct subjectivist 
interpretation of probability needs, given that arguably no grading of belief (as opposed to acceptance) can be represented by an additive measure.

2. Probability and randomness. Randomness has been proposed for a long time as the basis for a definition of probability. But the very idea of the randomness of a typical sequence of coin tosses needs clarification. Progress on this question leads us from reliance on a pragmatic notion of unpredictability to appeal to an epistemic notion of informational complexity: the fruitful contrast between the sequence $111111 \ldots$ and the coin tosses sequence lies in the higher information-content of the second one (its description is more complex). In the 1960's, several definitions of the complexity of sequences were proposed in terms of the minimal length of the programs producing them as outputs. Such definitions, that have been shown equivalent and that also satisfy strong properties of robustness (they are "machine-independant"), seem to provide an "absolute" analysis of our pre-systematic notion of randomness, so that the present situation of the randomness theory is comparable to the situation of the calculability theory in the 1930's, as Church and others provided their equivalent analysis of the pre-theoric notion of effective procedure. Jean-Paul Delahaye's paper is devoted to a systematic discussion of this comparison.

3. The management of uncertainty: probability theory and its rivals. A salient feature of the recent period in the philosophy of probability is the emergence of new, non-probabilistic ways to deal with uncertainty. As explained by Didier Dubois and Henri Prade in their comprehensive survey, several reasons contribute to the explanation of this emergence.

Firstly the very concept of probability has always been, as noticed by Hacking, a Janus-faced one: one statistical side, concerned with stochastic laws of chance processes, and an epistemic side, concerned with the measurement of the strength of the (reasonable) belief in sentences possibly devoid of any statistical content. The two notions, that remained quite unrelated until about 1660 , have been progressively thought-though at the price of many distortions-as intertranslatable and at the end unified as governed by the same laws, namely Kolmogorov's. The revival of an 
autonomous concept of epistemic probability in recent times is rooted in remarks and suggestions made by both philosophers (L.J. Cohen) and statisticians (G. Shafer) in the 1970's. According to the main suggestion, which leads to a noticeable departure from the standard probabilistic framework w.r.t. the combination rules that are used when several evidential items are available, favourable and unfavourable evidences are to be weighted separately: the 'epistemic probability' does not obey the rule of additivity. In Shafer's theory of evidence, for example, the axiom of additivity is replaced by a more general inequality: the probability $p$ of a proposition and the probability $q$ of its negation obey $0 \leq p \leq 1,0 \leq q$ $\leq 1$ and $p+q \leq 1$ occurs only when there is very strong evidence on both sides of the question, and we have both $p<1$ and $q=0$ if there is evidence for the proposition but none against it.

Difficulties with conditional logic are another major source of nonstandard probabilities. According to a well-known trivialization result due to Lewis, it is unsuitable to identify the probability of a conditional with the conditional probability of the consequent of the conditional given its antecedent. Adams tried however to maintain probabilistic semantics for conditionals, the leading idea of which was 'probabilistic soundness': it should not be possible for all premisses of an acceptable inference to be probable while the conclusion is improbable. This kind of soundness is generally out of reach to the naive way of building such semantics: for the so-called paradox of lottery shows that the uncertainties of the premisses, however small they are, can accumulate in an unfortunate way and at the end authorize maximally uncertain conclusions. Thus Adams proposes probabilistic semantics of a new kind-now termed ' $\varepsilon$-semantics'-in terms not of high probability, but of probability 'as high as necessary to ensure arbitrary high certainty in the conclusions'. These semantics have been shown roughly equivalent to possibilistic semantics developed by Dubois and Prade.

Wolfgang Spohn's system of natural conditional functions is another way of dealing with uncertainty in a non-probabilistic setting. Such conditional functions $x$, which are intended to represent epistemic states by grading disbelief in propositions, are related to Adams' logic by the equation $x(B / A)=n$ iff $P(B / A) \approx \varepsilon^{n}$ (with an infinitesimal $\varepsilon$ ). The aim of Spohn's paper is to account for the objective side of the causation 
relation by analysing it via conditional dependance relative to these conditional functions rather than relative to their probabilistic counterparts. The probabilistic approach of uncertainty remains however a very active field of research. Statisticians and computer scientists have elaborated several powerful algorithms able to cope with one of the biggest difficulties in this perspective, namely the high complexity of the computations. Jean-Louis Golmard's paper reviews the methods that are used in order to implement efficiently the probabilistic reasoning, especially those that are developed under the label 'bayesian networks'. 